\title{
Single-shot X-ray Dark-field Imaging
}

\section{Zhili Wang*, Dalin Liu, Kun Ren, Xiaomin Shi and Jianlin Xia}

School of Electronic Science \& Applied Physics, Hefei University of Technology, Hefei, People's Republic of China

*dywangzl@hfut.edu.cn

X-ray grating-based dark-field imaging have emerged as promising extensions to traditional attenuationbased radiography and computed tomography [1]. Particularly, the dark-field signal enables the detection of features below the spatial resolution limit of the imaging system [2]. In recent years, several potential applications of X-ray grating-based dark-field imaging have been explored, including preclinical applications [3], material science, and so on. Presently, the phase-stepping technique is routinely used to retrieve the dark-field signal [1]. However, in the limit of low photon counts, it has been shown the dark-field signal cannot be retrieved since the measurement of visibility follows a Rician distribution [4]. Therefore, there is a lower limit for the required photon number to successfully extract the dark-field signal using the phase-stepping technique. This implies that dose reduction could be difficult, which hinders the future clinical implementation of X-ray grating-based dark-field imaging. To overcome this limitation, a two-shot approach has been proposed to accurately retrieve the dark-field signal even in the case of very low photon statistics [5].

In this contribution, we report on a novel single-shot approach for dose-saving retrieval of dark-field signal in X-ray grating-based phase-contrast imaging. Most importantly, the dark-field signal can be accurately retrieved by this method even in the limit of low photon counts. As shown in Fig. 1, the novel method is based on manipulation of the relative shift in the dual-detector configuration. The first detector is located at the peak position of the intensity curve, i.e., $x_{g}^{1} / p_{2}=0$, while the second at the valley position, i.e., $x_{g}^{2} / p_{2}=1 / 2$. Under the reasonable assumption of a Gaussian scattering distribution, the two intensities by the detectors are respectively given by,

$$
\begin{aligned}
& I_{1}=T \cdot\left[1+V_{1} \cdot \exp \left(-\frac{2 \pi^{2}}{p^{2}} d_{1}^{2} \cdot D F\right)\right] \\
& I_{2}=T \cdot\left[1-V_{2} \cdot \exp \left(-\frac{2 \pi^{2}}{p^{2}} d_{2}^{2} \cdot D F\right)\right]
\end{aligned}
$$

where $T$ represents the sample's attenuation, $V_{1}$ and $V_{2}$ are the reference visbilities without the object, respectively, $p$ is the grating period, $d_{1}$ and $d_{2}$ are the distances downstream the phase grating G1 of the first and second detectors, respectively, and DF is the object's dark-field signal. 


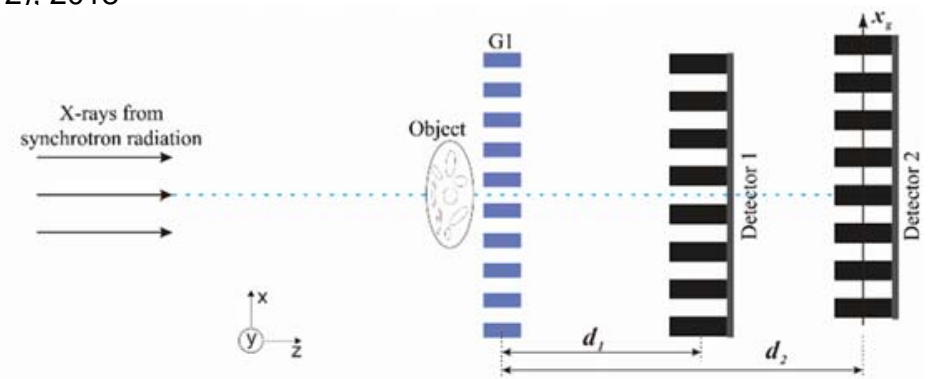

Figure. 1. Configuration of X-ray grating-based phase-contrast imaging using dual detectors. By combining the two intensity measurements, we can exclude the attenuation signal from:

$$
\frac{1-V_{2} \cdot \exp \left(-2 \pi^{2} d_{2}^{2} \cdot D F / p^{2}\right)}{1+V_{1} \cdot \exp \left(-2 \pi^{2} d_{1}^{2} \cdot D F / p^{2}\right)} \equiv F(D F)
$$

Note that since the dark-field signal can never be negative, the above combination limits the possible values of the function $F(D F)$ to the range [0;1]. As shown in Fig. 2, this function is always monotonously growing in the full region, and trends to saturation for large values of the DF signal. For the dark-field signal within this region, it can be uniquely retrieved from the single-valued inverse function,

$$
D F=F^{-1}\left[\frac{1-V_{2} \cdot \exp \left(-2 \pi^{2} d_{2}^{2} \cdot D F / p^{2}\right)}{1+V_{1} \cdot \exp \left(-2 \pi^{2} d_{1}^{2} \cdot D F / p^{2}\right)}\right]
$$

In comparison to the phase-stepping technique, the advantage of our method is twofold: firstly only a single object exposure is needed, and secondly correct retrieval of the dark-field signal is possible even for very short exposure times. Both implies a further dose reduction, which is a strict prerequisite for the clinical implementation of X-ray grating-based phase-contrast imaging.

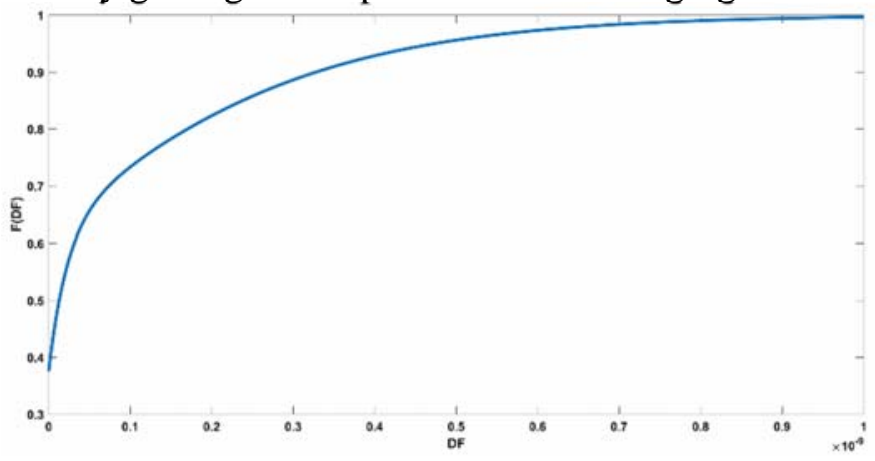

Figure. 2. Plot of the monotonic function $F(D F)$.

\section{References:}

[1] F Pfeiffer et al, Nat. Mater. 7 (2008) 134.

[2] W Yashiro et al, Opt. Express 18 (2010) 16890.

[3] A Bravin et al, Phys. Med. Biol. 58 (2013) R1.

[4] M Chabior et al, J. Appl. Phys. 110 (2011) 053105.

[5] M Marschner et al, Opt. Express 24 (2016) 27032.

[6] The authors acknowledge funding from the National Natural Science Foundation of China (11475170, U1532113, 11205157). 